Chapman University

Chapman University Digital Commons

$11-2021$

Kinematic Assessment of Turning and Walking Tasks Among

Stroke Survivors by Employing Wearable Sensors and Pressure

Platform

Masoud Abdollahi

Pranav Madhav Kuber

Christopher Hoang

Michael Shiraishi

Rahul Soangra

See next page for additional authors

Follow this and additional works at: https://digitalcommons.chapman.edu/pt_articles

Part of the Musculoskeletal System Commons, Nervous System Commons, Nervous System Diseases Commons, Other Rehabilitation and Therapy Commons, and the Physical Therapy Commons 


\section{Kinematic Assessment of Turning and Walking Tasks Among Stroke Survivors by Employing Wearable Sensors and Pressure Platform}

\section{Comments}

This is a pre-copy-editing, author-produced PDF of an article presented at the 2021 43rd Annual International Conference of the IEEE Engineering in Medicine \& Biology Society (EMBC). The definitive

publisher-authenticated version is available online at https://doi.org/10.1109/EMBC46164.2021.9630791

\section{Copyright}

(c) 2021 IEEE. Personal use of this material is permitted. Permission from IEEE must be obtained for all other uses, in any current or future media, including reprinting/republishing this material for advertising or promotional purposes, creating new collective works, for resale or redistribution to servers or lists, or reuse of any copyrighted component of this work in other works.

\section{Authors}

Masoud Abdollahi, Pranav Madhav Kuber, Christopher Hoang, Michael Shiraishi, Rahul Soangra, and Ehsan Rashedi 


\title{
Kinematic Assessment of Turning and Walking Tasks Among Stroke Survivors by Employing Wearable Sensors and Pressure Platform
}

\author{
Masoud Abdollahi, Pranav Madhav Kuber, Christopher Hoang, Michael Shiraishi, Rahul Soangra, \\ Ehsan Rashedi*
}

\begin{abstract}
Stroke survivors often experience reduced movement capabilities due to alterations in their neuromusculoskeletal systems. Modern sensor technologies and motion analyses can facilitate the determination of these changes. Our work aims to assess the potential of using wearable motion sensors to analyze the movement of stroke survivors and identifying the affected functions. We recruited 10 participants (5 stroke survivors, 5 healthy individuals) and conducted a controlled laboratory evaluation for two of the most common daily activities: turning and walking. Among the extracted kinematic parameters, range of trunk and sacrum lateral bending in turning were significantly larger in stroke survivors (p-value<0.02). However, no statistical difference in mean angular velocity and range of motion for trunk/sacrum/shank flexion-extension were obtained in the turning task. Our results also indicated that during walking, while there was no difference in swing time, double support portion of gait among the stroke group was significantly larger $(p$-value $=0.001)$. Outcomes of this investigation may help in designing new rehabilitation programs for stroke and other neurological disorders and/or in improving the efficacy of such programs.
\end{abstract}

Clinical Relevance - This study may provide a better insight on the detailed functional differences between stroke survivors and healthy individuals which in turn could be used to develop a more efficient rehabilitation program for stroke community.

\section{INTRODUCTION}

Neurological disorders (e.g., Alzheimer, stroke, epilepsy, and Parkinson's disease) and their consequences yet remain a critical problem from being fully understood, much less developing preventive strategies or even cure. This remains true even though humankind has evolved through generations of research and has led to increased longevity by the development of medication for several fatal diseases and disorders. On the other hand, disorders affecting neuromuscular function, which may or may not involve multiple systems (like muscular, neurological, and cognition, etc.) are even more complex. Looking at the immense nervous system composing of billions of neural links to every fiber of the body, even a slight change in the structural pathway of a neuron can result into altered function. One of the most common neurological diseases is Stroke, which has a

M. Abdollahi, P. M. Kuber, and E. Rashedi are with the Industrial and Systems Engineering Department, Rochester Institute of Technology, Rochester, NY, USA

C. Hoang and M. Shiraishi are with Department of Physical Therapy, Crean College of Health and Behavioral Sciences, Chapman University, Orange, CA, USA prevalence of $\sim 7$ million people in the U.S. with a high financial burden of $\sim 75$ billion USD, as reported in 2013 [1]. Among the several effects of such disorders include sensory dysfunction, paralysis, reduced muscle activity and cognition, and treatment may or may not result in full recovery. In such cases, the surviving patients undergo rehabilitation programs, which may be helpful in the recovery of lost functions.

Movement limitation is among the major effects of neurological disorders leading to a reduced stability and increased fall risk [2], [3]. Thus, it is critical to identify the changes in the performance and physical capability of the stroke survivors during activities of daily living (ADLs) to put them in the right path of physical recovery. One of the tools helpful in performance assessment can be motion analysis [4], [5]. For example, one prior study showed that motion systems can provide key insights on the changes in gait parameters after stroke [3]. Another research showed similar benefits for Parkinson's disease using wearable sensors during a walking task [6]. This shows that motion analysis can be implemented as a key information in functional recovery and/or monitoring the performance on individuals suffering from neurological disorders.

Although studies have been conducted on various ADLs, there is a lack of adequate focus on the turning tasks performed by stroke survivors [7], [8]. Besides the type of activity, current trends in the literature of human movement science show a translation from camera-based to wearable sensorbased motion analysis [9], [10]. A type of such wearable sensors is the Inertial Measurement Unit (IMU) sensors, which have not been implemented in assessment of turning in stroke community and can provide benefits such as portability, accessibility, and usability (e.g., by embedding them on garments). Through our work we have utilized techniques involving motion analysis by wearable sensors to evaluate the set of potentially affected parameters in $360^{\circ}$ turning, as well as walking. Another objective of this study was to explore the capability of IMUs in identifying the changes in stroke survivors' performance. The outcome of this study could have several applications such as designing efficient rehabilitation programs, developing a performance monitoring tool, and enhancing the insight about the effects of stroke on neuromusculoskeletal systems.

R. Soangra is with Department of Physical Therapy, Crean College of Health and Behavioral Sciences, Chapman University, Orange, CA, USA and Fowler School of Engineering, Chapman University, Orange, CA, USA.

* Please address all correspondence to E. Rashedi, Industrial and Systems Engineering Department, Rochester Institute of Technology, One Lomb Memorial Drive, Rochester, NY 14623, Phone: 585-475-7260, Email: exreie@rit.edu 


\section{Methodology}

\section{A. Participants}

A total of 10 subjects (5 Stroke survivors, 5 healthy individuals) were recruited in this study. The t-test analysis depicted no significant difference ( $\mathrm{p}$-value $<0.05$ ) on the anthropometric measures among healthy and stroke groups (Table 1). An informed consent form was signed by all the participants prior to study approved by Chapman university institutional review board. Inclusion criteria of the participants were that they should have been able to walk $>10 \mathrm{~m}$ without assistance, have no sign of cognitive impairment, and had stroke $>6$-months prior to participation.

TABLE I. ANTHROPOMETRIC MEASURES OF THE PARTICIPANTS

\begin{tabular}{cccc}
\hline Parameters & $\begin{array}{c}\text { Stroke } \\
\text { Survivors }\end{array}$ & $\begin{array}{c}\text { Healthy } \\
\text { Individuals }\end{array}$ & $\begin{array}{c}\boldsymbol{p} \text {-Value } \\
\text { (ANOVA) }\end{array}$ \\
\hline Gender & $\begin{array}{c}4 \text { males } \\
1 \text { female }\end{array}$ & $\begin{array}{c}3 \text { males } \\
2 \text { females }\end{array}$ & - \\
\hline Age (year) & $59.6(7.3)$ & $67.6(4.3)$ & 0.13 \\
\hline Height $(\mathrm{cm})$ & $180.3(7.6)$ & $171.7(10)$ & 0.22 \\
\hline Weight $(\mathrm{kg})$ & $98.9(22)$ & $80.5(14.1)$ & 0.23 \\
\hline
\end{tabular}

\section{B. Approach}

A set of 4 IMUs (XSens, Enschede, Netherlands) were placed on each of the shanks, sternum, and sacrum of the participant (Figure 1). These sensors captured the kinematics of the relevant segments while the participants performed the turning task with a sampling rate of $60 \mathrm{~Hz}$. Participants were told to stand straight, and an initial position was assigned along with a shoulder-level target in front of them. Then they were asked to turn 360 degrees in their normal fashion (Figure 1). The turning consisted of several cycles of lifting and touch down of the feet. Specifically, similar to gait we have heelstrike (HS) and toe-offs (TO) as the key events. One turning cycle would consist of stance and swing phases. The stance phase would begin with HS and end with TO while swing phase would start with TO and ends with HS. The subjects were instructed to look at the same target after turning 360 degrees. Meanwhile, pressure platform (GAITRite, New Jersey, USA) was used for gait analysis during walking task. For the walking task, the participants were instructed to walk in a straight path for $10 \mathrm{~m}$ in normal fashion on the pressure platform.

\section{Data Analysis}

A custom-designed code was developed for analyzing the data in MATLAB (MathWorks, Natick, MA, USA). Angle of segment, angular velocity, and linear acceleration captured by XSENS sensors were initially filtered using a Butterworth filter with a cut-off frequency of $5 \mathrm{~Hz}$. To analyze the turning process, it is essential to identify the HS and TO events. To determine the time of HS and TO, we used the angular velocity of the shank sensor.

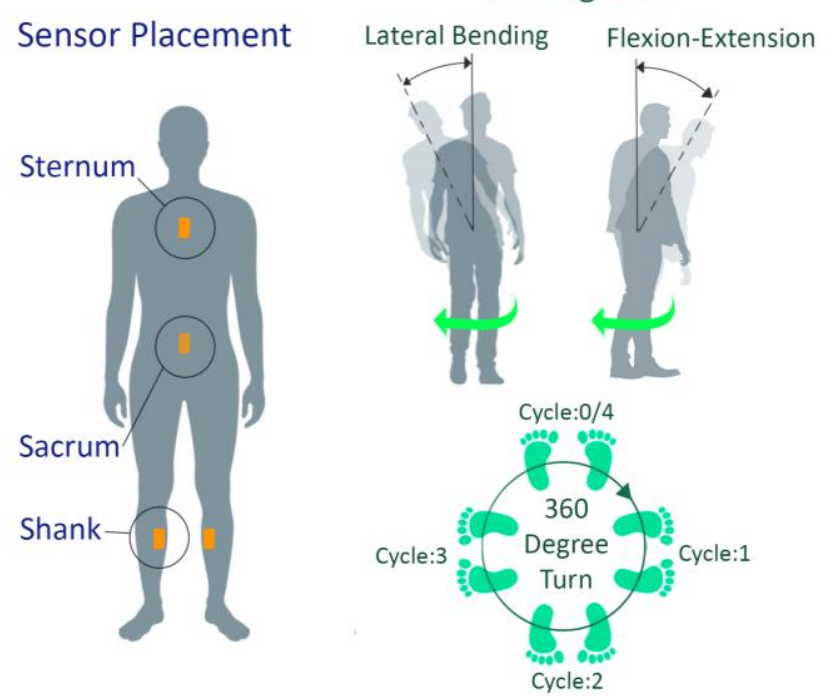

Figure 1. An illustration depicting the turning task along with the placement of wearable sensors for recording movement.

The signal in consideration (angular velocity of the sensor in the lateral direction of the shank) consisted of several peaks showing the maximum velocity in the swing phase of the shank. To find exact event of TO and HS, we identified the start of the rising trend to reach the peak, and the end of the falling trend after the peak respectively.

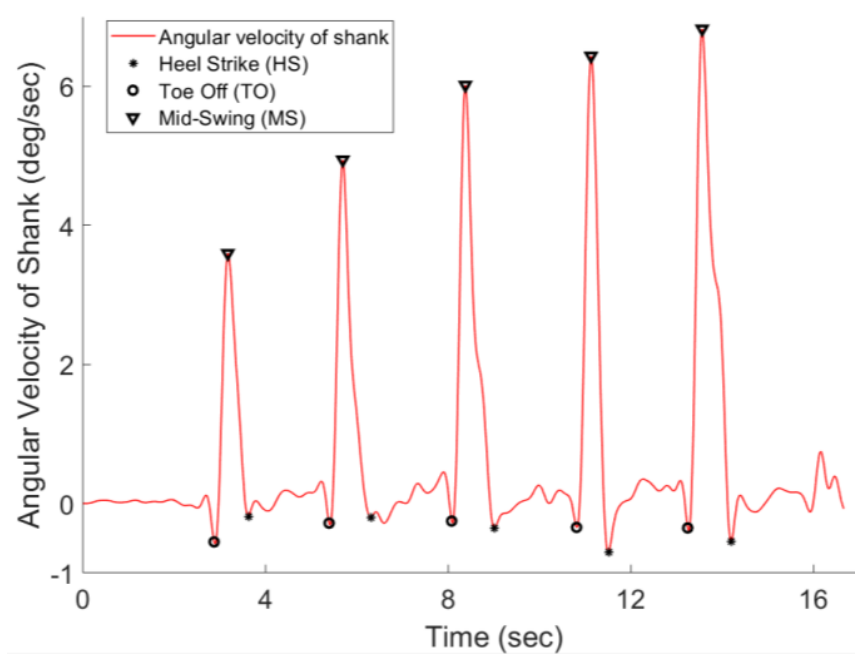

Figure 2: Angular velocity of shank: Detection and Segmentation

By determining the TO and HS events, we were able to segment the turning signals for all of the four IMU sensors. Eleven parameters representing various factors and kinematic variables were calculated to quantify the motion of turning. These included number of cycles, duration of turn, mean Angular velocity of flexion for trunk/shank/sacrum, ratio of stance time to duration of turn, range of trunk/sacrum lateral bending, range of sacrum/trunk/shank flexion-extension.

The GAITRite system directly provides the gait parameters which include velocity, cadence, swing time, stride length, stride velocity, double support in gait cycle $(\%)$, cycle time 
and stance percentage. The procedure for calculating the parameters was repeated for each participant and mean (S.D) values were reported. Next, we conducted a t-test for comparing the results for stroke survivors and healthy individuals for both the tasks.

\section{RESULTS}

The results of t-test analysis for turning showed stroke survivors had a greater number of cycles, duration of turn, range of trunk lateral bending, and range of sacrum lateral bending ( $p$-values $<0.05$, Table 2). Moreover, the ratio of stance time to duration of turn $(\%)$ in stroke survivors was greater than healthy individuals. However, the significance for this parameter was marginal.

TABLE II. STATISTICAL COMPARISON AND MEAN (SD) OF SPATIOTEMPORAL FEATURES FOR HEALTHY INDIVIDUALS AND STROKE SURVIVORS

\begin{tabular}{cccc}
\hline Feature Name & $\begin{array}{c}\text { Stroke } \\
\text { Survivors } \\
\text { Mean (SD) }\end{array}$ & $\begin{array}{c}\text { Healthy } \\
\text { Individuals } \\
\text { Mean (SD) }\end{array}$ & p-values \\
\hline Number of Cycles & $7.4(2.3)$ & $3.8(0.8)$ & $\mathbf{0 . 0 4 0}$ \\
\hline Duration of Turn (sec) & $9.6(3.5)$ & $4.4(1.9)$ & $\mathbf{0 . 0 4 1}$ \\
\hline $\begin{array}{c}\text { Mean Angular Velocity of } \\
\text { Trunk Flexion (deg/sec) }\end{array}$ & $0.3(0.8)$ & $1.1(0.2)$ & 0.156 \\
\hline $\begin{array}{c}\text { Mean Angular Velocity of } \\
\text { Sacrum Flexion (deg/sec) }\end{array}$ & $0.2(0.9)$ & $1.1(0.2)$ & 0.139 \\
\hline $\begin{array}{c}\text { Mean Angular Velocity of } \\
\text { Shank Flexion (deg/sec) }\end{array}$ & $1.6(0.5)$ & $2(0.3)$ & 0.153 \\
\hline $\begin{array}{c}\text { Ratio of Stance Time to } \\
\text { Duration of Turn (\%) }\end{array}$ & $57.9(16.6)$ & $42.9(7.6)$ & $\mathbf{0 . 0 5 4}$ \\
\hline $\begin{array}{c}\text { Range of Trunk Lateral } \\
\text { Bending (deg) }\end{array}$ & $12.7(4.8)$ & $5.8(1.0)$ & $\mathbf{0 . 0 1 7}$ \\
\hline $\begin{array}{c}\text { Range of Sacrum Lateral } \\
\text { Bending (deg) }\end{array}$ & $10.9(2.3)$ & $6.7(0.8)$ & $\mathbf{0 . 0 0 6}$ \\
\hline $\begin{array}{c}\text { Range of Trunk Flexion- } \\
\text { Extension (deg) }\end{array}$ & $9.5(2.9)$ & $6.5(1.9)$ & 0.110 \\
\hline $\begin{array}{c}\text { Range of Sacrum Flexion- } \\
\text { Extension (deg) }\end{array}$ & $7.7(3.8)$ & $6.9(0.9)$ & 0.613 \\
\hline $\begin{array}{c}\text { Range of Shank Flexion- } \\
\text { Extension (deg) }\end{array}$ & $17.7(8.1)$ & $11(1.8)$ & 0.179 \\
\hline
\end{tabular}

TABLE III. STATISTICAL COMPARISON AND MEAN (SD) OF SEVERAL GAIT PARAMETERS FOR HEALTHY INDIVIDUALS AND STROKE SURVIVORS

\begin{tabular}{cccc}
\hline \multirow{2}{*}{ Gait Parameter } & $\begin{array}{c}\text { Stroke } \\
\text { Survivors } \\
\text { Mean (SD) }\end{array}$ & $\begin{array}{c}\text { Healthy } \\
\text { Individuals } \\
\text { Mean (SD) }\end{array}$ & p-values \\
\hline Velocity (cm/s) & $85.9(20.6)$ & $113.3(22.8)$ & $\mathbf{0 . 0 0 7}$ \\
\hline Cadence (steps/min) & $100.3(8.3)$ & $111.7(12)$ & $\mathbf{0 . 0 1 8}$ \\
\hline Cycle Time (sec) & $1.2(0.1)$ & $1.1(0.1)$ & $\mathbf{0 . 0 3 1}$ \\
\hline Swing Time (sec) & $0.42(0.03)$ & $0.41(0.04)$ & 0.511 \\
\hline Stride Length (cm) & $102.3(20)$ & $121.1(13.6)$ & $\mathbf{0 . 0 1 8}$ \\
\hline Stride Velocity (cm/sec) & $86.38(21.1)$ & $113.3(22.8)$ & $\mathbf{0 . 0 0 8}$ \\
\hline Stance (\%) & $60.8(3.7)$ & $62.4(1.8)$ & 0.243 \\
\hline $\begin{array}{c}\text { Double Support in gait } \\
\text { cycle (\%) }\end{array}$ & $30.1(3.7)$ & $25.1(2.8)$ & $\mathbf{0 . 0 0 1}$ \\
\hline
\end{tabular}

Meanwhile, similar analysis for walking demonstrated that stroke survivors showed significantly lower velocity, cadence, stride length, stride velocity and higher values of cycle time and percentage of double support of gait cycle ( $p$ values $<0.04$, Table 3). A statistical test (KolmogorovSmirnov test) was conducted on all of the parameters for testing the normality of the data. The results of this analysis did not show any deviations from normality in our dataset.

\section{DISCUSSION}

In this study, movement limitation induced by stroke has been quantified by analyzing the kinematic data obtained from wearable IMU sensors and pressure platform. We calculated several parameters relating to the performance of individuals during 360 degree turning and walking. Our findings indicated that besides the traditionally measured pace of motion, other parameters such as trunk deflection in turning and portion of double support phase in gait cycle were significantly different between the participant groups.

Among the various motion capture systems, four IMU sensors were utilized in this study, which are more portable and user friendly compared to camera-based motion capture systems. In future, we will use these sensors for the walking task as well due to these mentioned features. Besides, as seen from the results, a single sensor can be used to identify the changes in movement of one body segment. Although using a single sensor instead of a four-sensor configuration can provide less information about whole body performance, it could further improve the usability and the potential for continues monitoring. In this case, our earlier study [11], demonstrated the possibility of replacing the IMU sensor with a smartphone which are also embedded with a motion sensor.

We considered a 360-degree turning as opposed to other commonly used turning tasks like Figure-of-eight and Timed Up and Go (TUG) [12], [13]. Unlike these tasks, a full turnaround does not involve acceleration/deceleration phases, which is the reason for selecting this activity. Based on the number of cycles, duration of turn and velocity of gait calculated in this study, stroke survivors, similar to patients suffering from Parkinson's disease [9], were slower in performing both turning and walking tasks. Interestingly, there was no significant difference between the mean angular velocity of flexion-extension in trunk, sacrum and shank (Table 2). However, range of lateral bending for trunk and sacrum were significantly different parameter between the participant groups. This means the stroke survivors had more trunk wobbling while turning. This extra movement in the stroke group could be related to the lack of proper core stability. Further, the wobbly motion in the lateral direction could indicate a lack of stability provided by the muscles responsible for the bending motion.

The range of flexion-extension angle for trunk, sacrum and shanks were not significantly different in the turning task. This means that the stroke survivors could perform the flexion-extension motion similar to healthy individuals. However, values of range of motion were considerably less (6.5, 6.9, 11 degrees for trunk, sacrum, and shank respectively) in the flexion-extension motion and could be the reason for not observing any significant difference. The ratio 
of stance time to duration of each turn cycle was significantly larger for the stroke group. This could be interpreted as the stroke survivors could not have normal duration of the swing phases due to lack of proper stability stemmed from their neuromusculoskeletal limitations.

Similar to the phases in the turning task, walking consists of iterative process of stance and swing phases. However, the observations depict that in contrast to turning, the ratio of stance to the whole cycle during walking was not a significant parameter (Table 3). The key reason for such variation could be the effect of turning motion on the vestibular system of the individuals, which is absent in walking. The chances of alterations in this system may be higher in stroke survivors, which can cause them to require more frequent support, as depicted by the higher values of the stance time percent in turning [14]. However, since full functionality of the vestibular system may not be utilized in walking tasks, stroke survivors showed similar values of stance time percentage as healthy individuals. On the other hand, double support portion in the gait cycle was significantly larger in stroke group. This could be justified by the fact that double support phase is the most stable phase of gait since two feet would be placed on the ground. By increasing the duration of this phase, stroke survivors could reduce the risk of fall and increase the overall stability during walking.

Few limitations can be recognized in the current study. The sample size needs to be increased in future for obtaining more power in statistical results. Using the pressure platform may reduce the accessibility/portability of our system. In the future, by replacing the pressure mat with IMU system, the accessibility of the system can be further enhanced. We have initiated this study with the presented nineteen parameters. However, in the future we would consider more parameters such as linear/angular acceleration of the segments. Moreover, even though this study consisted of determining changes in the movement of stroke survivors, the concept can have broader implications. For example, the identified parameters in the study can be used for the development of performance monitoring and/or fall risk assessment models for a variety of neurological disorders.

\section{CONCLUSION}

To provide a better insight about the effects of stroke on the physical performance of the stroke survivors, this study explored the capability of wearable sensors along with a pressure mat in analysis of the 360-degree turning and walking. The results of our work shed light upon the differences between the kinematic parameters which were significantly affected by a stroke. We found that core stability plays important role in the task of turning. The outcome of this study could facilitate development of targeted rehabilitation programs and performance monitoring tools.

\section{REFERENCES}

[1] C. L. Gooch, E. Pracht, and A. R. Borenstein, "The burden of neurological disease in the United States: A summary report and call to action," Ann. Neurol., vol. 81, no. 4, pp. 479-484, 2017.

[2] A. Mirelman et al., "Gait impairments in Parkinson's disease," Lancet Neurol., vol. 18, no. 7, pp. 697-708, 2019.

[3] K. K. Patterson, W. H. Gage, D. Brooks, S. E. Black, and W. E. McIlroy, "Changes in gait symmetry and velocity after stroke: A cross-sectional study from weeks to years after stroke," Neurorehabil. Neural Repair, vol. 24, no. 9, pp. 783-790, 2010.

[4] J. M. Czerniecki, "Rehabilitation in limb deficiency. 1. Gait and motion analysis," Arch. Phys. Med. Rehabil., vol. 77, no. 3 SUPPL., 1996.

[5] R. Soangra, V. Krishnan, J. John, E. Rashedi, and A. McKenzie, "Comparison of $360^{\circ}$ Turn Cycles among Individuals after Stroke and Healthy Older Adults," Appl. Sci., vol. 11, no. 7, p. 3202, 2021.

[6] J. Bae, K. Kong, N. Byl, and M. Tomizuka, "A mobile gait monitoring system for abnormal gait diagnosis and rehabilitation: A pilot study for parkinson disease patients," J. Biomech. Eng., vol. 133, no. 4, pp. 1-11, 2011.

[7] D. A. Nowak, "The impact of stroke on the performance of grasping: Usefulness of kinetic and kinematic motion analysis," Neurosci. Biobehav. Rev., vol. 32, no. 8, pp. 1439-1450, 2008.

[8] Y. R. Mao et al., "The crucial changes of sit-to-stand phases in subacute stroke survivors identified by movement decomposition analysis," Front. Neurol., vol. 9, no. MAR, pp. 1-8, 2018.

[9] M. Son et al., "Evaluation of the turning characteristics according to the severity of Parkinson disease during the timed up and go test," Aging Clin. Exp. Res., vol. 29, no. 6, pp. 1191-1199, 2017.

[10] L. Haertner et al., "Effect of fear of falling on turning performance in Parkinson's disease in the lab and at home," Front. Aging Neurosci., vol. 10, no. MAR, pp. 18, 2018.

[11] S. Karvekar, M. Abdollahi, and E. Rashedi, "Smartphonebased human fatigue level detection using machine learning approaches," Ergonomics, vol. 64, no. 5, pp. 600612, 2020.

[12] S. S. T. Wong, M. S. Yam, and S. S. M. Ng, "The Figureof-Eight Walk test: Reliability and associations with stroke-specific impairments," Disabil. Rehabil., vol. 35, no. 22, pp. 1896-1902, 2013.

[13] C. U. Persson, A. Danielsson, K. S. Sunnerhagen, A. Grimby-Ekman, and P.-O. Hansson, "Timed Up \& Go as a measure for longitudinal change in mobility after stroke Postural Stroke Study in Gothenburg (POSTGOT)," $J$. Neuroeng. Rehabil., vol. 11, no. 1, pp. 1-7, 2014.

[14] B. I. Han, H. S. Song, and J. S. Kim, "Vestibular rehabilitation therapy: Review of indications, mechanisms, and key exercises," J. Clin. Neurol., vol. 7, no. 4, pp. 184196, 2011. 